\title{
Erythrokeratodermia variabilis: A new case
}

\section{Fatima Zahra Elfatoiki, Sofia Capatas, Hayat Dahbi Skali, Fouzia Hali, Soumia Chiheb}

\author{
Department of Dermatology, Ibn Rochd UHC, 1 quartier des Hopitaux 20000, Casablanca, Morocco
}

Corresponding author: Dr. Fatima Zahra Elfatoiki, E-mail: Fatiza59@hotmail.fr

\begin{abstract}
Erythrokeratodermia variabilis is a rare genodermatosis associated with keratinisation disorders. We report a case of erythrokeratodermia variabilis in Moroccan infant. A 22 -month-old girl was referred to our department with reddishbrown hyperkeratotic and well-demarcated plaques on the extremities. The lesions started when he was 4 months of age. The patient was treated by emollients and keratolytic cream. Erythrokeratodermia variabilis is characterized by the association of fixed well-demarcated plaques and transient erythematous patches. Although cutaneous histopathology is not specific, a typical physical clinical feature can aid the diagnosis.
\end{abstract}

Key words: Erythrokeratodermia variabilis; Genodermatosis; Disorder; Keratinisation; Connexins 


\title{
Erythrokératodermie variable: Un nouveau cas
}

\section{Fatima Zahra Elfatoiki, Sofia Capatas, Hayat Dahbi Skali, Fouzia Hali, Soumia Chiheb}

\author{
Department of Dermatology, Ibn Rochd UHC, 1 quartier des Hopitaux 20000, Casablanca, Morocco
}

Corresponding author: Dr. Fatima Zahra Elfatoiki, E-mail: Fatiza59@hotmail.fr

\begin{abstract}
RÉSUMÉ
L'érythrokératodermie variable est une génodermatose rare due à un trouble de la kératinisation. Nous rapportons un cas d'érythrokératodermie variable chez un nourrisson marocain. Une fille de 22 mois avait consulté pour des placards erythémato-sqameux pigmentés hyperkératosiques bien limités des membres évoluant depuis l’âge de 4 mois. L'aspect clinique était en faveur d'une érythrokeratodermie variable. La patiente était traitée par émollients et kératolytiques. Lérythrokératodermie variable est caractérisée par un aspect clinique typique qui permet de poser le diagnostic malgré l'aspect histopathologique non spécifique.
\end{abstract}

Mots clé: Erythrokératodermie variable; Génodermatose; Trouble; Kératinisation; Connexines

\section{INTRODUCTION}

L'érythrokératodermie variable est une maladie génétique à expression cutanée liée à une mutation des gènes codant pour les connexines. Nous rapportons le cas d'une érythrokératodermie variable chez un nourrisson de 22 mois.

\section{OBSERVATION}

Nourrisson d'origine marocaine, âgée de 22 mois, née de parents non consanguins. A été vue en consultation pour des lésions diffuses prurigineuses des membres évoluant depuis l'âge de 4 mois. Lexamen dermatologique retrouvait une xérose cutanée importante, des placards erythématosqameux pigmentés hyperkératosiques bien limités. Ces lésions avaient une disposition relativement symétrique et siégeaient sur les quatre membres (Figs. 1 et 2). Il n'existait pas d'atteinte muqueuse ou phanérienne. Le reste de l'examen clinique était sans particularité. Le tableau clinique était compatible avec une erytrokératodermie variable. La confirmation génétique n’a pas été proposée par manque de moyens. Un traitement topique par émollients et kératolytiques (urée à 5\%) a été proposé.

\section{DISCUSSION}

L'erytrokératodermie variable est une genodermatose rare. Son mode de transmission est le plus souvent autosomique dominant avec des mutations portant sur des gènes codant pour les connexines 31 et 30.3. Les connexines sont des protéines transmembranaires qui participent à la formation des jonctions GAP. Celles-ci interviennent dans les communications intercellulaires de presque tous les tissus dont la peau $[1,2]$.

L'expression clinique est variable. Les lésions apparaissent le plus souvent lors de la première année de vie. Lerytrokératodermie variable se caractérise par deux types de lésions; des placards eryhthématosquameux pigmentés hyperkératosiques bien limités persistants classiquement symétriques et des plaques erythémateuses irrégulières fugaces. Les lésions prédominent sur le tronc et les membres [1].

L'histologie des lésions cutanées reste peu spécifique. Cependant, en cas de réalisation de biopsie cutanée, l'étude histologique peut trouver dans l'épiderme une hyperkératose ortho- ou parakératosique, une acanthose et une papillomatose en faveur du diagnostic de l'erytrokératodermie variable. Dans le derme, une dilatation capillaire et un infiltrat

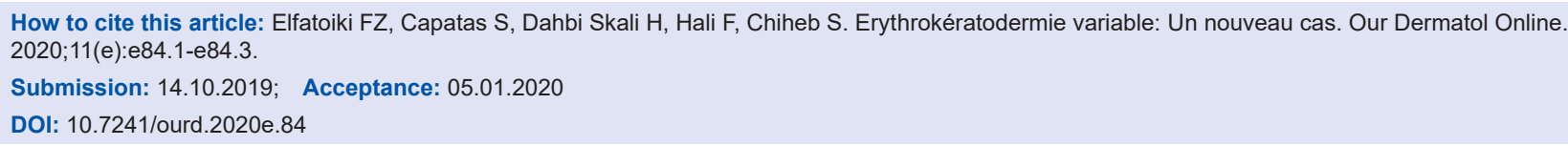




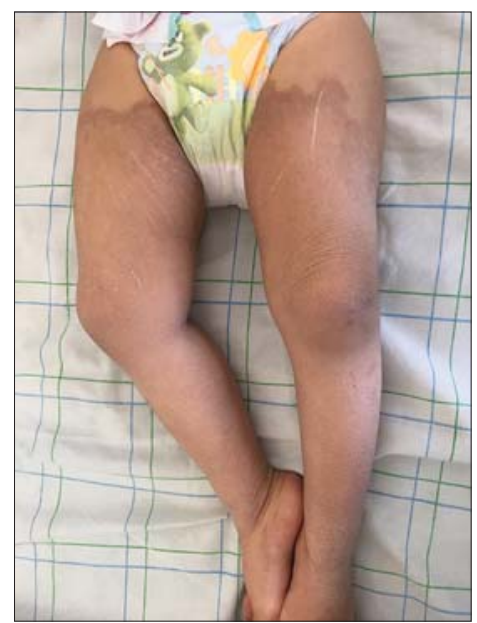

Figura 1: Placards erythémato-sqameux pigmentés bien limités des membres inferieurs.

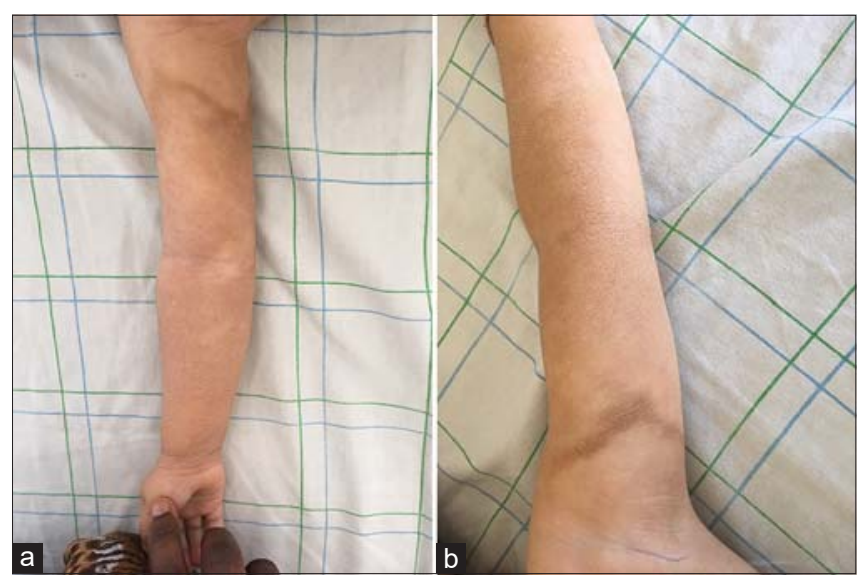

Figura 2: $(a$ and $b)$ Placard erythémato-sqameux pigmenté bien limité du membre supérieur droit et du membre supérieur gauche.

lymphocytaire périvasculaire modéré peuvent être observés [1].

Le diagnostic différentiel de l'erytrokératodermie variable se pose essentiellement avec l'érythrokératodermie variable et progressive caractérisée par des plaques érythémateuses et hyperkératosiques plus diffuses et symétriques des extrémités et de la face. Il a même été suggéré que les deux génodermatoses soient deux variantes phénotypiques d'une même maladie et que l'érythrokératodermie variable et progressive soit la variante sévère de la maladie [3].

Le traitement de l'erytrokératodermie variable reste symptomatique. Les traitements topiques consistent en des émollients et des kératolytiques. Les rétinoïdes par voie générale, acitrétine ou isotrétinoïne à faibles doses et au long cours, peuvent permettre une amélioration des lésions hyperkératosiques [1].

En conclusion, l'erytrokératodermie variable est une maladie génétique à expression purement cutanée. Son tableau clinique est très caractéristique. Létude génétique à la recherche des mutations des gènes codant pour les connexines permet la confirmation du diagnostic. Son traitement de référence repose sur les rétinoïdes par voie générale.

\section{Consent}

The examination of the patient was conducted according to the Declaration of Helsinki principles.

The authors certify that they have obtained all appropriate patient consent forms. In the form the patient(s) has/have given his/her/ their consent for his/her/their images and other clinical information to be reported in the journal. The patients understand that their names and initials will not be published and due efforts will be made to conceal their identity, but anonymity cannot be guaranteed.

\section{RÉFÉRENCES}

1. Ajebo E, Wall WB, Davis LS. Chronic symmetrically distributed hyperpigmented plaques in a middle-age woman. JAAD Case Rep. 2019;5:249-51.

2. Karadag AS, Bilgili SG, Calka O, Bayram I. Erythrokeratodermia variabilis: Two case reports. Indian Derm Online J. 2013;4:340.

3. Guo BR, Cai HB, Zong WK, Li CS, Liu LZ, Qiao S, et al. A heterozygous mutation in GJA1 gene in Chinese family with serious erythrokeratodermia variabilis et progressive. Chin Med J (Engl). 2019;132:86-8.

Copyright by Fatima Zahra Elfatoiki, et al. This is an open-access article distributed under the terms of the Creative Commons Attribution License, which permits unrestricted use, distribution, and reproduction in any medium, provided the original author and source are credited.

Source of Support: Nil, Conflict of Interest: None declared. 